\title{
TRABALHAR COM IDOSOS: FATORES ASSOCIADOS AO INTERESSE DE ESTUDANTES DE ENFERMAGEM NO BRASIL E NO CHILE
}

\author{
WORKING WITH THE ELDERLY: FACTORS \\ ASSOCIATED WITH THE INTEREST OF NURSING \\ STUDENTS IN BRAZIL AND CHILE
}

\section{TRABAJAR CON ANCIANOS: FACTORES ASOCIADOS CON EL INTERÉS DE LOS ESTUDIANTES DE ENFERMERÍA EN BRASIL Y CHILE}

\author{
Rosana Maria Barreto Colichi ${ }^{1}$ \\ Andres Eduardo Jimenez Figueroa ${ }^{2}$ \\ Verónica Gómez Urrutia ${ }^{3}$ \\ Hélio Rubens de Carvalho Nunes ${ }^{4}$ \\ Silvana Andrea Molina Lima ${ }^{5}$
}

\begin{abstract}
Como citar este artigo: Colichi RMB, Figueroa AEJ, Urrutia VG, Nunes HRC, Lima SAM. Trabalhar com idosos: fatores associados ao interesse de estudantes de enfermagem no Brasil e no Chile. Rev baiana enferm. 2021;35:e42429.

Objetivo: identificar fatores associados ao interesse em trabalhar com idosos como futuro mercado de trabalho entre estudantes de enfermagem do Brasil e do Chile. Método: estudo transversal analítico, realizado entre março e setembro de 2018, incluindo 885 estudantes de enfermagem. Utilizou-se formulário com informações sociodemográficas, relacionadas à família e áreas de atuação e o Inventário de Percepção de Suporte Familiar. A análise estatística incluiu associações bivariadas e modelos de regressão logística múltipla. Resultados: 27,5\% dos estudantes consideraram trabalhar com idosos como possível atuação profissional, sem associação com variáveis familiares. Verificaram-se associações positivas entre aqueles que cursavam em instituições públicas e citavam trabalhar com crianças e associações negativas entre alunos mais velhos. Conclusão: para a maioria dos estudantes de enfermagem do Brasil e do Chile, o trabalho com idosos não era considerado como possibilidade de atuação profissional; essa possibilidade era menor entre os alunos mais velhos e maior entre alunos de instituições públicas.
\end{abstract}

Descritores: Envelhecimento. Estudantes de Enfermagem. Educação em Enfermagem. Mercado de Trabalho. Intenção.

Objective: to identify factors associated with the interest in working with the elderly as a future job market among nursing students from Brazil and Chile. Method: cross-sectional analytical study, conducted between March and September 2018, including 885 nursing students. A form was used with sociodemographic information related to

\footnotetext{
Administradora. Mestre em Enfermagem. Universidade Estadual Paulista Júlio de Mesquita Filho. Botucatu, São Paulo, Brasil. rosana.barreto-colichi@unesp.br. https://orcid.org/0000-0002-8765-3965.

2 Psicólogo Organizacional. Doutor em Administração de Empresas. Professor da Facultad de Psicologia, Universidad de Talca. Talca, Chile. https://orcid.org/0000$0001-8324-0250$

3 Jornalista. Doutora em Sociologia e Estudos de Gênero. Professora da Facultad de Ciencias Sociales y Humanidades da Universidad Autónoma de Chile. Talca, Chile. https://orcid.org/0000-0002-2399-7566.

${ }^{4}$ Estatístico. Doutor em Saúde Coletiva. Professor da Universidade Estadual Paulista Júlio de Mesquita Filho. Botucatu, São Paulo, Brasil. https://orcid.org/0000-00027806-1386.

5 Enfermeira. Doutora em Ginecologia, Obstetrícia e Mastologia. Professora da Universidade Estadual Paulista Júlio de Mesquita Filho. Botucatu, São Paulo, Brasil.
} https://orcid.org/0000-0001-9945-2928. 
family and areas of activity and the Family Support Perception Inventory. Statistical analysis included bivariate associations and multiple logistic regression models. Results: $27.5 \%$ of the students considered working with the elderly as a possible professional performance, without association with family variables. There were positive associations between those who attended public institutions and cited working with children and negative associations among older students. Conclusion: for most nursing students in Brazil and Chile, working with the elderly was not considered as a possibility of professional performance; this possibility was lower among older students and higher among students from public institutions.

Descriptors: Aging. Nursing Students. Nursing Education. Labor Market. Intention.

Objetivo: identificar factores asociados con el interés en trabajar con los ancianos como un futuro mercado laboral entre los estudiantes de enfermería de Brasil y Chile. Método: estudio analítico transversal, realizado entre marzo y septiembre de 2018, incluyendo 885 estudiantes de enfermería. Se utilizó un formulario con información sociodemográfica relacionada con la familia y las áreas de actividad y el Inventario de Percepción de Apoyo Familiar. El análisis estadístico incluyó asociaciones bivariantes y múltiples modelos de regresión logística. Resultados: El $27,5 \%$ de los estudiantes consideró trabajar con los ancianos como un posible desempeño profesional, sin asociación con variables familiares. Hubo asociaciones positivas entre estudiantes de instituciones públicas y citaron trabajar con niños y asociaciones negativas entre los estudiantes mayores. Conclusión: para la mayoría de los estudiantes de enfermería en Brasil y Chile, el trabajo con los ancianos no fue considerado como una posibilidad de desempeño profesional; esta posibilidad fue menor entre los estudiantes mayores y más grande entre los estudiantes de instituciones públicas.

Descriptores: Envejecimiento. Estudiantes de Enfermería. Educación en Enfermería. Mercado laboral. Intención.

\section{Introdução}

Acompanhando as tendências globais de envelhecimento populacional, o Brasil e o Chile apresentam dados preocupantes. No Brasil, há projeções de aumento da expectativa de vida ao nascer em 2030 para 78,64 $\operatorname{anos}^{(1)}$. As pessoas com mais de 65 anos de idade que, no ano 2020, representavam 9,83\% passarão a representar 13,54\% da população, em 2030, e 25,49\% em $2060^{(1)}$. No Chile, a expectativa de vida em 2020 estava estimada em 85,25 $\operatorname{anos}^{(2)}$. Dados de 2017 já indicavam uma projeção de pessoas idosas (com 60 anos ou mais) correspondente a 19,3\% da população ${ }^{(3)}$. Assim, em números absolutos, o número de pessoas idosas em 2021 aproxima-se de 22 milhões no Brasil ${ }^{(1)}$ e 3,5 milhões no Chile ${ }^{(2)}$.

Os impactos econômicos desse envelhecimento podem ser percebidos na forma de aumento da demanda de serviços especializados, na fabricação de produtos fármaco-químicos, farmacêuticos, estéticos, nutricionais, ampliação da saúde privada, intermediação financeira, seguros, previdência complementar, entre outros ${ }^{(4)}$.

$\mathrm{Na}$ área da saúde, os reflexos do envelhecimento populacional na América Latina vêm sendo amplamente observados na demanda de cuidado dos idosos, retratados principalmente pela dependência funcional, numa proporção crescente, de acordo com o aumento da idade. Uma meta-análise revelou que há alta prevalência de incapacidade funcional em idosos brasileiros, sendo a média igual a 42,8\% $( \pm 21,0)$ entre as mulheres e $39,6 \%( \pm 26,2)$ entre os homens $^{(5)}$. No Chile, $14,2 \%$ dos idosos apresentam alguma dependência funcional, necessitando de auxílio para realização de atividades da vida diária (AVD), tais como comer, banhar-se, mover-se dentro de casa, utilizar o banheiro, deitar ou levantar da cama ou vestir-se ${ }^{(2)}$.

Formas de financiamento e disponibilização de serviços de saúde para essa população, por meio de modelos de atenção focados na promoção e na prevenção da saúde, evitaria a sobrecarga do sistema de saúde ${ }^{(4,6)}$. Entretanto, apesar de iniciativas como o Examen de Medicina Preventiva del Aulto Mayor (EMPAM) no Chile, as atuais políticas públicas latino-americanas mostram-se insuficientes, não acompanhando as transformações demográficas e sociais ${ }^{(7)}$.

Além disso, o cuidado ao idoso, que antes era provido informalmente pelos familiares, tem sofrido alterações pelas novas dinâmicas sociais 
e novos arranjos familiares, que incluem muitos indivíduos optando por manterem-se sozinhos, a monoparentalidade, a homoparentalidade, casais que optam por não ter filhos, entre outros. Junto com a inserção da mulher no mercado de trabalho, as mudanças sociais afetarão cada vez mais o cuidado informal dos idosos ${ }^{(8)}$.

Há uma tendência, portanto, de haver lacunas no futuro acerca do cuidado e da saúde do idoso, abrindo campos de atuação ao profissional enfermeiro. Desse modo, os programas de educação têm a responsabilidade de preparar os futuros enfermeiros, para que ultrapassem a visão restrita da saúde e incluam intervenções socioculturais numa participação ativa com os idosos, com o objetivo de melhorar sua qualidade de vida ${ }^{(9)}$.

No entanto, estudo de revisão ${ }^{(10)}$ sobre o preparo de enfermeiros e estudantes de enfermagem para cuidar de idosos em países de baixa e média renda revelou, além do conhecimento insuficiente e de concepções errôneas sobre o cuidado de idosos, a baixa preferência por trabalhar com esses indivíduos.

Pesquisas internacionais apontam, no futuro profissional de enfermagem, distanciamento do desejo de cuidar de idosos, revelando motivações relacionadas às questões culturais e carência de aprofundamento do tema durante a graduação. Fatores demográficos ou sociais, como relacionamento familiar, convivência com idosos fora do ambiente hospitalar, etnia e religião ${ }^{(11-12)}$, poderiam também impactar nas escolhas de carreira e influenciar no desejo dos estudantes em trabalhar com idosos ${ }^{(13)}$.

Apesar da relevância do tema, observa-se a escassez de pesquisas na América Latina. Conhecer o interesse de estudantes de enfermagem em trabalhar com idosos no futuro profissional, como possibilidade de mercado de trabalho, em países como o Brasil e o Chile, pode ajudar a orientar o desenvolvimento de programas relacionados ao envelhecimento nesses locais, numa atitude inovadora fundamentada na economia da longevidade, garantindo sustentabilidade de serviços de enfermagem para a crescente população idosa.
Assim, o presente estudo teve por objetivo identificar fatores associados ao interesse em trabalhar com idosos como futuro mercado de trabalho entre estudantes de enfermagem do Brasil e do Chile.

\section{Método}

Este é um estudo transversal analítico, norteado pela ferramenta STROBE, com dados coletados de março a setembro de 2018 em cinco instituições de ensino brasileiras e chilenas. No Brasil, participaram alunos de curso diurno de uma universidade pública e de dois cursos noturnos de instituições particulares do interior do estado de São Paulo. No Chile, tomaram parte estudantes de cursos diurnos de uma universidade pública e uma privada, ambas da região de Maule. Os cursos de enfermagem abrangiam o período de cinco anos, com exceção da universidade pública brasileira ( 4 anos).

Com população total de 1.196 estudantes inscritos nos cursos de graduação de Enfermagem, a amostra não probabilística (intencional) abrangeu $74 \%(n=885)$ de respondentes em relação à população.

O instrumento de coleta de dados, projetado em português e espanhol pelos pesquisadores, foi composto de duas partes: questionário com informações sociodemográficas e relacionadas à família e à renda; planilha para preenchimento de respostas abertas para citação de possibilidades de áreas de atuação em relação ao mercado de trabalho do futuro enfermeiro.

Para avaliar o trabalho com idosos como possibilidade de mercado de trabalho, as palavras citadas pelos entrevistados na segunda parte do instrumento de coleta foram examinadas em busca de termos relacionados ao envelhecimento ou idoso, tais como: geriatria, gerontologia, casas de repouso, Instituições de Longa Permanência de Idosos (ILPI), cuidador de idosos entre outros.

A fim de verificar as relações familiares dos entrevistados, foi também utilizado o Inventário de Percepção de Suporte Familiar (IPSF). Este é um instrumento brasileiro, validado no Chile ${ }^{(14)}$ e tem boa consistência interna $(0,93)$. Composto 
por 42 itens, serve para avaliar três fatores: consistência afetiva (expresso pelos relacionamentos afetivos positivos dentro da família, de interesses para o outro, expressão verbal e não verbal de carinho, clareza nos papéis e regras dos membros da família, bem como habilidades e estratégias para enfrentar situações de conflito); adaptação familiar (verificado pelas expressões de sentimentos negativos sobre a família, tais como exclusão, raiva, vergonha, relações agressivas, irritação, mal-entendido, competência percebida na família, interesses e culpa entre os membros da família, em situações de conflito); e autonomia familiar (percepção de relacionamentos de confiança, privacidade e liberdade entre os membros da família) ${ }^{(15)}$.

Com procedimento de abordagem padronizado nos dois países, os alunos foram convidados verbalmente pelos pesquisadores, no horário das aulas, sendo explicitada a participação voluntária no estudo. O preenchimento dos instrumentos levou cerca de 30 minutos, sendo recolhidos e cifrados para reconhecimento apenas da instituição de ensino e país.

A análise dos fatores associados com o interesse em trabalhar com idosos após a conclusão da graduação em enfermagem foi realizada ajustando modelos de regressão logística múltipla. Associações foram consideradas estatisticamente significativas se $\mathrm{p}<0,05$. A análise foi feita com o software SPSS v21.0.

Aspectos éticos foram respeitados nos dois países. No Brasil, o estudo foi previamente aprovado pelo Comitê de Ética em Pesquisa da Faculdade de Medicina de Botucatu da Universidade Estadual Paulista (UNESP), pelos Pareceres 2.499.340 e 2.885.923; no Chile, o projeto que inclui esta pesquisa foi aprovado pelo Comité de Ética da Universidad Autónoma de Chile pela Acta de Evaluación n. 75-18. Os participantes do estudo assinaram o Termo de Consentimento Livre e Esclarecido.

\section{Resultados}

Participaram da pesquisa 378 estudantes brasileiros (43,7\%) e 487 chilenos (56,3\%), totalizando uma amostra de 865 alunos, composta, em sua maioria, por mulheres $(81,5 \%)$, vinda de instituições privadas $(55,1 \%)$ com idade entre 20 e 25 anos (60,2\%), conforme Tabela 1.

Com relação às informações familiares, a Tabela 1 mostra que o maior contingente mora com a família $(76,2 \%)$, não tem filhos $(87,7 \%)$ nem companheiros $(88,9 \%)$ e possui renda familiar mais baixa $(38,1 \%)$. A pontuação do suporte familiar percebido considerou a mediana de $63(\min =0 ; \max =82$ ) no Brasil e de 65 ( $\min =0$; $\max =80$ ) no Chile.

A Tabela 1 mostra ainda, com relação às pretensões para o futuro profissional após a graduação, que menos de um terço dos alunos aponta possibilidades de trabalho relacionadas a idosos, tanto no Brasil ( $\mathrm{n}=117 ; 31,0 \%)$ como no Chile ( $\mathrm{n}=121 ; 24,8 \%)$.

Tabela 1 - Distribuição das variáveis sociodemográficas dos estudantes, informações familiares, visão de possibilidade de carreira relacionada ao idoso. Brasil e Chile - 2018. ( $\mathrm{N}=865)$

(continua)

\begin{tabular}{l|c|c|c|c|c|c}
\hline \multirow{2}{*}{ Variáveis } & \multicolumn{2}{|c|}{ Brasil } & \multicolumn{2}{c|}{ Chile } & \multicolumn{2}{c}{ Total } \\
\cline { 2 - 7 } Tipo de instituição & $\mathbf{n ~ ( 3 7 8 )}$ & $\mathbf{0}$ & $\mathbf{n ~ ( 4 8 7 )}$ & $\mathbf{\%}$ & $\mathbf{n ~ ( 8 6 5 )}$ & $\mathbf{\%}$ \\
$\quad$ Pública & 106 & 28,0 & 282 & 57,9 & 388 & 44,9 \\
$\quad$ Privada & 272 & 72,0 & 205 & 42,1 & 477 & 55,1 \\
Ano do curso & & & & & & \\
1 & 89 & 23,5 & 80 & 16,4 & 169 & 19,5 \\
2 & 95 & 25,1 & 128 & 26,3 & 223 & 25,8 \\
3 & 102 & 27,0 & 112 & 23,0 & 214 & 24,7 \\
4 & 87 & 23,0 & 105 & 21,6 & 192 & 22,2 \\
5 & 5 & 1,3 & 62 & 12,7 & 67 & 7,7
\end{tabular}


Tabela 1 - Distribuição das variáveis sociodemográficas dos estudantes, informações familiares, visão de possibilidade de carreira relacionada ao idoso. Brasil e Chile -2018 . ( $\mathrm{N}=865)$ (conclusão)

\begin{tabular}{|c|c|c|c|c|c|c|}
\hline \multirow{2}{*}{ Variáveis } & \multicolumn{2}{|c|}{ Brasil } & \multicolumn{2}{|c|}{ Chile } & \multicolumn{2}{|c|}{ Total } \\
\hline & n (378) & $\%$ & n (487) & $\%$ & n (865) & $\%$ \\
\hline \multicolumn{7}{|l|}{ Sexo } \\
\hline Feminino & 333 & 88,1 & 372 & 76,4 & 705 & 81,5 \\
\hline Masculino & 45 & 11,9 & 115 & 23,6 & 160 & 18,5 \\
\hline \multicolumn{7}{|l|}{ Faixa etária } \\
\hline$<=19$ & 97 & 25,7 & 94 & 19,3 & 191 & 22,1 \\
\hline $20-25$ & 171 & 45,2 & 350 & 71,9 & 521 & 60,2 \\
\hline 26 ou mais & 110 & 29,1 & 43 & 8,8 & 153 & 17,7 \\
\hline \multicolumn{7}{|l|}{ Estado civil } \\
\hline Sem companheiro & 288 & 76,2 & 481 & 98,8 & 769 & 88,9 \\
\hline Com companheiro & 90 & 23,8 & 6 & 1,2 & 96 & 11,1 \\
\hline \multicolumn{7}{|l|}{ Tem filho } \\
\hline Não & 296 & 78,3 & 463 & 95,1 & 759 & 87,7 \\
\hline Sim & 82 & 21,7 & 24 & 4,9 & 106 & 12,3 \\
\hline \multicolumn{7}{|l|}{ Renda familiar } \\
\hline Faixa 1 & 178 & 47,1 & 97 & 19,9 & 275 & 31,8 \\
\hline Faixa 2 & 147 & 38,9 & 106 & 21,8 & 253 & 29,2 \\
\hline Faixa 3 & 34 & 9,0 & 112 & 23,0 & 146 & 16,9 \\
\hline Faixa 4 & 9 & 2,4 & 96 & 19,7 & 105 & 12,1 \\
\hline Faixa 5 & 10 & 2,6 & 76 & 15,6 & 86 & 9,9 \\
\hline \multicolumn{7}{|l|}{ Mora com a família } \\
\hline Não & 101 & 26,7 & 105 & 21,6 & 206 & 23,8 \\
\hline Sim & 277 & 73,3 & 382 & 78,4 & 659 & 76,2 \\
\hline \multicolumn{7}{|l|}{ Mora com alguém } \\
\hline Não & 366 & 96,8 & 397 & 81,5 & 763 & 88,2 \\
\hline Sim & 12 & 3,2 & 90 & 18,5 & 102 & 11,8 \\
\hline \multicolumn{7}{|l|}{ Mora só } \\
\hline Não & 347 & 91,8 & 474 & 97,3 & 821 & 94,9 \\
\hline Sim & 31 & 8,2 & 13 & 2,7 & 44 & 5,1 \\
\hline $\begin{array}{l}\text { Inventário de Percepção de } \\
\text { Suporte Familiar (mediana) }\end{array}$ & 63 & & 65 & & & \\
\hline \multicolumn{7}{|l|}{ Intenção de empreender } \\
\hline Não & 355 & 93,9 & 455 & 93,4 & 810 & 93,6 \\
\hline Sim & 23 & 6,1 & 32 & 6,6 & 55 & 6,4 \\
\hline \multicolumn{7}{|c|}{ Trabalhar com crianças no futuro } \\
\hline Não & 228 & 60,3 & 356 & 73,1 & 584 & 67,5 \\
\hline $\operatorname{Sim}$ & 150 & 39,7 & 131 & 26,9 & 281 & 32,5 \\
\hline \multicolumn{7}{|c|}{ Trabalhar com idosos no futuro } \\
\hline Não & 261 & 69,0 & 366 & 75,2 & 627 & 72,5 \\
\hline Sim & 117 & 31,0 & 121 & 24,8 & 238 & 27,5 \\
\hline
\end{tabular}

Fonte: Elaboração própria.

Pelo modelo de associações bivariadas descritas na Tabela 2, diferenças significativas foram observadas nos fatores que poderiam condicionar essa intenção, sendo maiores no Brasil em relação ao Chile ( $\mathrm{p}=0,046$; OR 0,74). No Chile, a chance de ter intenção de trabalhar com idosos é menor entre alunos de instituições privadas
( $\mathrm{p}<0,001$; OR 0,38$)$, da mesma forma que é menor entre alunos de 20 a 25 anos em comparação aos alunos mais jovens ( $\mathrm{p}=0,018$; OR 0,65$)$. Por outro lado, a possibilidade de trabalhar com idoso aumenta no Chile entre aqueles que moram com alguém ( $\mathrm{p}=0,040$; OR 1,68), enquanto, no Brasil, essa chance ocorre entre aqueles que moram 
sozinhos $(\mathrm{p}=0,032$; OR 1,07). Além disso, considerar trabalhar com idosos foi maior entre alunos que veem também a possibilidade de trabalhar com crianças no futuro no Brasil ( $\mathrm{p}<0,001$; OR $3,37)$ e no Chile $(p<0,001 ;$ OR 6,86). Não foram encontradas associações nos dois países entre nenhuma das variáveis familiares estudadas, tais como estado civil, ter filhos, morar com a família ou suporte familiar percebido.

Tabela 2-Associações bivariadas para a chance de considerar a possibilidade de trabalhar com idosos como futuro mercado de trabalho. Brasil e Chile - 2018. (N=865)

\begin{tabular}{|c|c|c|c|c|c|c|c|c|}
\hline \multirow{3}{*}{$\begin{array}{l}\text { Variáveis } \\
\text { Tipo de instituição }\end{array}$} & \multicolumn{4}{|c|}{ Brasil } & \multicolumn{4}{|c|}{ Chile } \\
\hline & \multirow{2}{*}{$\begin{array}{c}\begin{array}{c}\text { Odds } \\
\text { Ratio }\end{array} \\
1,19\end{array}$} & \multicolumn{2}{|c|}{$\begin{array}{c}\text { Intervalo de } \\
\text { Confiança } \\
95 \% \\
\end{array}$} & \multirow{2}{*}{$\begin{array}{c}\text { p-value } \\
0,487\end{array}$} & \multirow{2}{*}{$\begin{array}{c}\text { Odds } \\
\text { Ratio }\end{array} \mid \begin{array}{c} \\
0,38\end{array}$} & \multicolumn{2}{|c|}{$\begin{array}{c}\text { Intervalo de } \\
\text { Confiança } \\
95 \% \\
\end{array}$} & \multirow{2}{*}{$\frac{\text { p-value }}{}$} \\
\hline & & 0,73 & 1,95 & & & 0,24 & 0,60 & \\
\hline Ano do curso & 0,86 & 0,71 & 1,04 & 0,125 & 0,91 & 0,77 & 1,07 & 0,239 \\
\hline Sexo & 0,79 & 0,39 & 1,59 & 0,508 & 0,65 & 0,39 & 1,10 & 0,106 \\
\hline Faixa etária (Referência $\leq$ 19anos) & & & & 0,594 & & & & 0,030 \\
\hline $20-25$ & 0,77 & 0,45 & 1,30 & 0,325 & 0,60 & 0,37 & 0,99 & 0,044 \\
\hline$\geq 26$ & 0,79 & 0,44 & 1,42 & 0,439 & 0,31 & 0,12 & 0,82 & 0,018 \\
\hline Estado civil & 1,08 & 0,65 & 1,80 & 0,765 & 1,52 & 0,28 & 8,41 & 0,631 \\
\hline Com filho & 1,29 & 0,77 & 2,17 & 0,329 & 0,79 & 0,29 & 2,16 & 0,642 \\
\hline Renda familiar & & & & 0,500 & & & & 0,590 \\
\hline Faixa 1 & 0,89 & 0,56 & 1,42 & 0,626 & 0,80 & 0,41 & 1,54 & 0,498 \\
\hline Faixa 2 & 0,52 & 0,22 & 1,27 & 0,152 & 1,01 & 0,54 & 1,90 & 0,966 \\
\hline Faixa 3 & 1,61 & 0,42 & 6,23 & 0,488 & 0,96 & 0,50 & 1,85 & 0,899 \\
\hline Faixa 4 & 0,50 & 0,10 & 2,45 & 0,396 & 1,40 & 0,72 & 2,74 & 0,320 \\
\hline $\begin{array}{l}\text { Inventário de Percepção de } \\
\text { Suporte Familiar }\end{array}$ & 1,00 & 0,99 & 1,01 & 0,926 & 1,00 & 0,99 & 1,02 & 0,638 \\
\hline Morar com família & 0,90 & 0,55 & 1,46 & 0,662 & 0,74 & 0,45 & 1,19 & 0,211 \\
\hline Morar com alguém & 1,12 & 0,33 & 3,79 & 0,856 & 1,68 & 1,02 & 2,76 & 0,040 \\
\hline Morar sozinho & 2,25 & 1,07 & 4,73 & 0,032 & 0,25 & 0,03 & 1,91 & 0,180 \\
\hline Intenção de ter negócio próprio & 0,78 & 0,30 & 2,02 & 0,603 & 1,41 & 0,65 & 3,07 & 0,388 \\
\hline Intenção cuidar de crianças & 3,37 & 2,14 & 5,30 & 0,000 & 6,86 & 4,36 & 10,80 & 0,000 \\
\hline
\end{tabular}

Fonte: Elaboração própria.

Quando utilizados modelos de regressão múltipla na amostra total, encontraram-se diferenças significativas apenas em relação às instituições públicas e privadas e ao cuidado de idosos e crianças. Assim, a chance de ter a intenção de trabalhar com idoso foi menor entre alunos de instituição privada ( $\mathrm{p}=0,009$; OR 0,58). Por outro lado, a chance de ter a intenção de trabalhar com idoso foi quase cinco vezes maior entre alunos que têm a intenção de trabalhar com crianças ( $\mathrm{p}<0,001$; OR 4,65).

Tabela 3 - Regressão múltipla para chance de considerar a possibilidade de trabalhar com idosos como futuro mercado de trabalho. Brasil e Chile -2018 . ( $\mathrm{N}=865)$

(continua)

\begin{tabular}{|c|c|c|c|c|}
\hline Variável & Odds Ratio & Intervalo & fiança & p-value \\
\hline Aluno do Chile $($ Referência $=$ Brasil $)$ & 0,72 & 0,47 & 1,09 & 0,117 \\
\hline Instituição Privada (Referência = Pública) & 0,58 & 0,38 & 0,87 & 0,009 \\
\hline Ano & 0,88 & 0,75 & 1,04 & 0,133 \\
\hline Sexo masculino & 0,85 & 0,55 & 1,33 & 0,490 \\
\hline Faixa etária (Ref $\leq 19$ anos) & & & & 0,847 \\
\hline $20-25$ & 1,05 & 0,65 & 1,68 & 0,841 \\
\hline$\geq 26$ & 1,18 & 0,65 & 2,16 & 0,585 \\
\hline
\end{tabular}


Tabela 3 - Regressão múltipla para chance de considerar a possibilidade de trabalhar com idosos como futuro mercado de trabalho. Brasil e Chile -2018 . $(\mathrm{N}=865)$

(conclusão)

\begin{tabular}{l|c|cc|c}
\hline Variável & Odds Ratio & Intervalo de Confiança & p-value \\
\hline Mora com a família & 1,63 & 0,76 & 3,51 & 0,209 \\
Mora com alguém & 2,41 & 0,99 & 5,83 & 0,052 \\
Mora só & 2,35 & 0,90 & 6,14 & 0,080 \\
Possui intenção de trabalhar com criança & 4,65 & 3,35 & 6,47 & 0,000 \\
\hline
\end{tabular}

Fonte: Elaboração própria.

\section{Discussão}

Este é o primeiro estudo que avalia o trabalho com idosos como possível mercado de atuação profissional dos futuros enfermeiros, comparando os dois países da América Latina e considerando questões familiares, além de incorporar potenciais fatores associados. Os resultados revelaram que, para a maioria dos estudantes de enfermagem entrevistados, o trabalho com idosos não era considerado como possível mercado de atuação profissional, sem associação com nenhuma das variáveis familiares estudadas. Sendo um pouco maior entre aqueles que cursavam em instituições públicas, no Chile, havia uma diminuição dessa possibilidade entre os alunos mais velhos em comparação aos mais jovens (até 19 anos). Por outro lado, a possibilidade aumentava no Chile entre aqueles que moravam com alguém enquanto, no Brasil, essa chance ocorria entre aqueles que moravam sozinhos. Era maior também entre alunos que citavam a possibilidade de trabalhar com crianças, nos dois países.

Com níveis abaixo do esperado, a maioria dos estudantes não considerava a possibilidade de trabalhar com idosos, confirmando o apontado por estudo ${ }^{(10)}$ sobre a baixa preferência em países de média e baixa renda. Este estudo contraria resultados de pesquisas realizadas em países desenvolvidos, como a Suécia, onde 58\% dos entrevistados responderam que consideravam trabalhar com idosos ${ }^{(16)}$, ou ainda Austrália, China e Estados Unidos, onde se verificaram atitudes favoráveis e percepção positiva de trabalhar com pessoas mais velhas ${ }^{(17-19)}$. No entanto, estudos da Turquia e Arábia Saudita, apesar de apresentarem médias individuais logo acima das pontuações neutras ou moderadas, elas também foram inferiores ao esperado, dada a importância cultural do envelhecimento nessas sociedades ${ }^{(11,20)}$. Esses achados podem indicar uma necessidade de promover as competências e habilidades inerentes ao cuidado do idoso, por meio de projetos pedagógicos apropriados que incentivem o trabalho futuro com essa população.

A despeito de nossas expectativas, nenhuma das variáveis familiares associou-se à expectativa de trabalhar com idosos, corroborando estudo realizado com 468 estudantes turcos, no qual os únicos preditores foram as atitudes acerca do envelhecimento ${ }^{(20)}$. Além disso, as atitudes dos alunos entrevistados naquela pesquisa eram piores entre aqueles que tinham um ancião morando com eles, indicando que a exposição a idosos não necessariamente melhorava as atitudes.

Pesquisa em Taiwan apontou que a disposição dos estudantes de enfermagem em cuidar de idosos não estava associada ao período de tempo com idosos ou avós que prestaram assistência na infância dos alunos, mas, sim, às atitudes dos estudantes em relação aos idosos, prestar atenção às questões relacionadas aos idosos e ter a experiência de ser voluntário no atendimento a idosos ${ }^{(13)}$. Isso indica que a participação em atividades educacionais em ambientes de cuidado de idosos, os conhecimentos sobre o envelhecimento e o próprio ambiente institucional que promova esse contato podem contribuir positivamente nas intenções de alunos em trabalhar futuramente com esses indivíduos.

Ainda que os estudantes brasileiros considerassem um pouco mais a possibilidade 
de trabalhar com idosos que os chilenos, não foram observadas diferenças significativas entre os dois países latinos. Tais achados podem estar associados à semelhança do contexto do histórico relacionado às suas emancipações políticas e características sociais e culturais, refletido no processo de desenvolvimento humano desses países $^{(3)}$. Neste mesmo sentido, pesquisa realizada entre estudantes de enfermagem da Coreia e dos Estados Unidos mostrou atitude dos coreanos mais negativa em relação aos adultos mais velhos, enquanto os estudantes americanos mostraram maior disposição para cuidar deles, provavelmente devido às grandes diferenças culturais $^{(19)}$.

A associação positiva entre a possibilidade de trabalhar com idoso e aqueles que cursavam em instituições públicas, mais expressiva no Chile, corrobora resultado de estudo de âmbito nacional realizado com 1.462 estudantes de enfermagem da Malásia, que também revelou que os alunos de ambientes educacionais públicos estão mais inclinados a cuidar de pessoas mais velhas, sendo exploradas questões religiosas e étnicas ${ }^{(12)}$. Esses achados podem revelar diferenças no perfil dos alunos que cursavam em instituições públicas e privadas nos dois países latinos. Convém ainda a reflexão acerca dos diferentes currículos adotados, já que as horas e o volume de estágio e sua inserção em locais de cuidado que incluíam o idoso podiam estar influenciando nesses resultados. No Brasil, alunos de escolas públicas moravam frequentemente sozinhos, já que grande parte provinha de outras cidades e frequentavam curso de período integral, apontando outra congruência nas associações encontradas no estudo.

Sobre a diminuição da possibilidade de atuação profissional junto a idosos entre os alunos mais velhos em comparação aos mais jovens (até 19 anos), esta pesquisa encontra similaridade com estudo da Turquia, em que, quanto mais velho o aluno, pior era a atitude perante o envelhecimento ${ }^{(20)}$, assim como os estudantes de enfermagem dos EUA, que mostraram baixa vontade de cuidar de idosos quando estavam no último ano ${ }^{(19)}$. Em pesquisas realizadas na Arábia Saudita, Taiwan e Suécia não se encontrou associação entre a idade e a disposição dos estudantes de trabalhar no cuidado ao idoso ${ }^{(11,13,16)}$. No entanto, estudo australiano com 886 estudantes de enfermagem demonstrou que os participantes mais jovens e os que não tinham experiência com idosos tinham percepções menos positivas em relação ao trabalho com esses indivíduos ${ }^{(17)}$. O estudo constatou que, apesar de os estudantes terem percepções positivas em relação ao trabalho com idosos, eles não quiseram comprometer-se a trabalhar com pessoas mais velhas quando se qualificassem ${ }^{(17)}$. Assim como apontado na investigação australiana ${ }^{(17)}$, esta pesquisa indica que os estudantes podem ter perdido o interesse pelo cuidado de idosos durante o curso, tendo desenvolvido maior aspiração por outros campos do cuidado de enfermagem, à medida que avançavam durante o programa. Pode também apontar diferenças geracionais entre os jovens estudados ${ }^{(8)}$. Resta compreender os motivos desse desinteresse em futuras pesquisas, a fim de atuar no restabelecimento e comprometimento no cuidado ao idoso.

Emerge, portanto, a necessidade de rever os planos de estudos, de forma que se adaptem ao atual contexto de trabalho e desenvolvimento profissional que os jovens millennials estão seguindo na enfermagem. Há que se considerar modificações nos perfis de graduação, de forma que sejam congruentes com as demandas do mundo do trabalho, tão influenciadas pelo envelhecimento populacional, bem como as expectativas em relação a esses jovens ${ }^{(21)}$, de modo que considerem, com maior destaque, as oportunidades de trabalho que os estudantes de enfermagem têm em outras áreas, como gestão, educação, pesquisa e empreendedorismo ${ }^{(22)}$.

Apontar a possibilidade de trabalho com idosos foi cinco vezes maior entre alunos que também citaram o trabalho com crianças. Isso pode refletir as premissas do cuidado em enfermagem relacionadas à humanização, independentemente da idade, e aos pilares da educação, apoiadas na história da profissão.

$\mathrm{Na}$ preparação para o aumento exponencial projetado do número de idosos, mudanças curriculares são muito necessárias em todas 
as ciências da saúde ${ }^{(20)}$. Considerando a baixa disponibilidade para trabalho com idosos apurado neste estudo realizado no Brasil e no Chile, torna-se essencial desenvolver habilidades e atitudes positivas em relação aos adultos mais velhos e o desejo de cuidado de idosos como possibilidade de mercado de trabalho dos futuros profissionais de enfermagem ${ }^{(10)}$.

Pesquisas confirmam a importância da preparação educacional e o ambiente de aprendizado favorável $^{(16,20)}$. Enfatizam ainda a relevância de educadores de enfermagem em reconhecer as atitudes positivas dos estudantes com base em práticas clínicas precoces no atendimento a idosos. Experiências de aprendizado direcionadas ao atendimento de idosos em uma variedade de contextos clínicos ${ }^{(16)}$, acompanhadas de enfermeiras especializadas em cuidados aos idosos $^{(17)}$, precisam ser implementadas nos currículos de enfermagem, garantindo que atitudes positivas prevaleçam durante todo o curso de enfermagem.

Desse modo, é necessário levar em consideração as necessidades e prioridades dessa geração de alunos, com suas novas trajetórias de trabalho e família. Nesse sentido, oportunizar a esses jovens essa experiência tem duplo sentido. Por um lado, favorece suas perspectivas; por outro lado, contribui para o desenvolvimento econômico, a coesão social e o bem-estar geral ${ }^{(23)}$. Adequar diretrizes curriculares para esse novo cenário está em consonância com o proposto por estudo ${ }^{(8)}$ que propõe a criação de políticas voltadas para esses jovens como forma de investimento no novo capital humano, promovendo a inserção laboral em empregos de qualidade, um tema muito polêmico para essa geração.

A amostra não ter sido probabilística é uma limitação deste estudo, mas a quase totalidade dos alunos de cada instituição participou da pesquisa, aumentando, assim, a possibilidade de os resultados serem generalizados para as instituições participantes. No entanto, há ressalvas e deve-se ter cuidado em generalizar os resultados, devido ao tamanho dessas duas populações. Ainda assim, o estudo traz importantes contribuições, ao discutir a importância de adequação do ensino de enfermagem, visando o preparo dos futuros enfermeiros e o pleno atendimento à população idosa.

\section{Conclusão}

O estudo revela que, para a maioria dos estudantes de enfermagem entrevistados no Brasil e no Chile, o trabalho com idosos não era considerado como possibilidade de atuação profissional; essa possibilidade era menor entre alunos mais velhos e maior entre alunos de instituições públicas. Entre aqueles era também citada a possibilidade de trabalhar com crianças.

Pesquisas futuras devem orientar o desenvolvimento de projetos pedagógicos na graduação de enfermagem que acentuem a inclusão do tema envelhecimento, a fim de garantir a oferta de serviços para a crescente população idosa na América Latina. Ao mesmo tempo, devem ampliar as possibilidades de atuação no mercado de trabalho dos futuros profissionais de enfermagem. Isso, certamente, trará reflexos positivos no atendimento às necessidades dos idosos, em uma sociedade que os reconheça e os agregue ao desenvolvimento sustentado, numa melhoria constante dos diferentes aspectos relacionados com a qualidade de vida e o bem-estar de todos.

\section{Colaborações:}

1 - concepção, projeto, análise e interpretação dos dados: Rosana Maria Barreto Colichi, Andres Eduardo Jimenez Figueroa, Verónica Gómez Urrutia e Hélio Rubens de Carvalho Nunes;

2 - redação do artigo e revisão crítica relevante do conteúdo intelectual: Rosana Maria Barreto Colichi, Andres Eduardo Jimenez Figueroa, Verónica Gómez Urrutia, Hélio Rubens de Carvalho Nunes e Silvana Andrea Molina Lima;

3 - aprovação final da versão a ser publicada: Rosana Maria Barreto Colichi, Andres Eduardo Jimenez Figueroa, Verónica Gómez Urrutia, Hélio Rubens de Carvalho Nunes e Silvan Andrea Molina Lima. 


\section{Referências}

1. Instituto Brasileiro de Geografia e Estatística. Projeções da População do Brasil e Unidades da Federação por sexo e idade: 2010-2060 [Internet]. Rio de Janeiro; 2018 [cited 2020 Nov 17]. Available from: https://www.ibge.gov.br/estatisticas/ sociais/populacao/9109-projecao-da-populacao. html?=\&t=resultados

2. Chile. Ministerio de Desarrollo Social y Familia. Adultos Mayores - Síntesis de Resultados, CASEN [Internet]. Santiago; 2017. [cited 2020 Nov 17]. Available from: http://observatorio. ministeriodesarrollosocial.gob.cl/storage/ docs/casen/2017/Resultados_Adulto_Mayores_ casen_2017.pdf

3. Santos CB, Pilatti LA, Pedroso B, Carvalho DR, Guimarães AM. Previsão do Índice de Desenvolvimento Humano e da expectativa de vida nos países da América Latina por meio de técnicas de mineração de dados. Ciênc saúde coletiva. 2018;23(11):3745-56. DOI: https://doi. org/10.1590/1413-812320182311.26142016

4. Oliveira ATR. Envelhecimento populacional e políticas públicas: desafios para o Brasil no século XXI. Espaço e Economia. 2016;(8):1-20. DOI: https://doi.org/10.4000/espacoeconomia.2140

5. Campos ACV, Almeida MHM, Campos GV, Bogutchi TF. Prevalence of functional incapacity by gender in elderly people in Brazil: a systematic review with meta-analysis. Rev bras geriatr gerontol. 2016;19(3):545-59. DOI: https://doi. org/10.1590/1809-98232016019.150086

6. Veras RP, Oliveira M. Envelhecer no Brasil: a construção de um modelo de cuidado. Ciênc saúde coletiva. 2018;23(6):1929-36. DOI: http:// doi.org/10.1590/1413-81232018236.04722018

7. Miranda GMD, Mendes ACG, Silva ALA. Population aging in Brazil: current and future social challenges and consequences. Rev bras geriatr gerontol. 2016;19(3):507-19. DOI: http:// dx.doi.org/10.1590/1809-98232016019.150140

8. Gómez-Urrutia V, Royo UP, Cruz CMA. Imagining Families: Gender, Youth, and Diversity in Chile. J Women Soc Work. 2017;32(4):491-503. DOI: https://doi.org/10.1177\%2F0886109917718232

9. Poblete-Troncoso M, Piña-Morán M. Enfermería gerontológica: desafíos para la promoción de los roles sociales en las personas mayores. Gerokomos [Internet]. 2019 [cited 2020 Sep
28];30(3):130-2. Available from: http://scielo.isciii. es/scielo.php?script=sci_arttext\&pid=S1134-928X2 019000300130\&lng=es\&nrm=iso

10. Abudu-Birresborn D, McCleary L, Puts M, Yakong V, Cranley L. Preparing nurses and nursing students to care for older adults in lower and middle-income countries: A scoping review. Int J Nurs Studies. 2019;92:121-34. DOI: https:// doi.org/10.1016/j.ijnurstu.2019.01.018

11. Alshehry AS, Almazan JU, Alquwez N. Infuence of Religiosity on the Saudi Nursing Students' Attitudes Toward Older People and Perceptions on Elderly Care. J Relig Health. 2019;59(6):2701-14. DOI: https://doi.org/10.1007/s10943-019-00857-z

12. Che CC, Chong MC, Hairi NN. What influences student nurses' intention to work with older people? A cross-sectional study. Int J Nurs Stud. 2018;85:61-7. DOI: 10.1016/j.ijnurstu.2018.05.007

13. Chi MJ, Shyu ML, Wang SY, Chuang HC, Chuang YH. Nursing Students' Willingness to Care for Older Adults in Taiwan. J Nurs Scholarsh. 2016;48:172-8. DOI: https://doi.org/10.1111/jnu. 12197

14. Jimenez-Figueroa AE, Mendiburo Subiabre NP, Olmedo Fuentes PA. Satisfacción familiar, apoyo familiar y conflicto trabajo-familia en una muestra de trabajadores chilenos. Av Psicol Latinoam [Internet]. 2011 [cited 2020 Sep 25];29(2):317-29. Available from: http://www. scielo.org.co/scielo.php?script $=$ sci_arttext\& pid=S1794- $47242011000200011 \& \operatorname{lng}=e n \& n r m=$ iso

15. Baptista MN. Inventário de percepção de suporte familiar (IPSF): estudo componencial em duas configurações. Psicol ciênc prof. 2007;27(3):496-509. DOI: 10.1590/S1414-9893200 7000300010

16. Carlson E, Idvall E. Who wants to work with older people? Swedish student nurses' willingness to work in elderly care-a questionnaire study. Nurse Educ Today. 2015;35(7):849-53. DOI: 10.1016/j.nedt.2015.03.002

17. Neville C. A cross-sectional view of Australian undergraduate nurses' perceptions of working with older people. Collegian. 2016;23(3):285-92. DOI: https://doi.org/10.1016/j.colegn.2015.05.003

18. Zhang S, Liu Y-H, Zhang H-F, Meng L-N, Liu P-X. Determinants of undergraduate nursing students' care willingness towards the elderly in China: Attitudes, gratitude and knowledge. 
Nurse Educ Today. 2016;43:28-33. DOI: 10.1016/ j.nedt.2016.04.021

19. Jang I, Oh D, Kim YS. Factors associated with nursing students' willingness to care for older adults in Korea and the United States. Int J Nurs Sci. 2019;6(4):426-31. DOI: 10.1016/j. ijnss.2019.09.004

20. Darling R, Sendir M, Atav S, Buyukyilmaz F. Undergraduate nursing students and the elderly: An assessment of attitudes in a Turkish university. Gerontol Geriatr Educ. 2018;39(3):283-94. DOI: https://doi.org/10.1080/02701960.2017.1311883

21. Escobar D, Covarrubias E. Expectativas académicas y laborales en estudiantes de enfermería.: Academic and labor expectations for nursing students. Rev cienc cuidad.
2019;16(2):59-71. DOI: https://doi.org/10.22463/ 17949831.1608

22. Medina E, Kaempffer AM. Medicina y otras Carreras de la Salud en Chile: Un análisis preliminar. Rev méd Chile. 2007;135(10):1346-54. DOI: http://dx.doi.org/10.4067/S0034-98872007 001000018

23. Novella R, Repetto A, Robino C, Rucci G, editores. Millennials en América Latina y el Caribe: ¿trabajar o estudiar? Washington, D.C.: Banco Interamericano de Desarollo; 2018. DOI: http:// dx.doi.org/10.18235/0001411

Recebido: 17 de novembro de 2020 Aprovado: 8 de março de 2021

Publicado: 13 de abril de 2021

A Revista Baiana de Enfermagem utiliza a Licença Creative Commons - Atribuição-NãoComercial 4.0 Internacional. https://creativecommons.org/licenses/by-nc/4.0/

Este artigo é de acesso aberto distribuído sob os termos da Licença Creative Commons (CC BY-NC). Esta licença permite que outros remixem, adaptem e criem a partir do seu trabalho para fins não comerciais. Embora os novos trabalhos tenham de lhe atribuir o devido crédito e não possam ser usados para fins comerciais, os usuários não têm de licenciar esses trabalhos derivados sob os mesmos termos. 